\title{
Matrix Metalloproteinase-dependent turnover of cartilage, synovial membrane, and connective tissue is elevated in rats with collagen induced arthritis
}

Anne Sofie Siebuhr ${ }^{1 *}$, Jianxia Wang ${ }^{2}$, Morten Karsdal' ${ }^{1}$, Anne-C Bay-Jensen ${ }^{1}$, Jin $\mathrm{Y}^{3}$ and Zheng $\mathrm{Q}^{4}$

\begin{abstract}
Background: Rheumatoid arthritis is a disease affecting the extracellular matrix of especially synovial joints. The thickness of the synovial membrane increases and surrounding tissue degrades, leading to altered collagen balance in the tissues. In this study, we investigated the altered tissue balance of cartilage, synovial membrane, and connective tissue in collagen induced arthritis (CIA) in rats.

Methods: Six newly developed ELISAs quantifying MMP-derived collagen degradation (C1M, C2M, and C3M) and formation (P1NP, P2NP, and P3NP) was used to detect cartilage turnover in rats with CIA. Moreover, CTX-II was used to detect alternative type II collagen degradation and as control of the model. 10 Lewis rats were injected with porcrine type II collagen twice with a 7 day interval and 10 rats was injected with $0.05 \mathrm{M}$ acetic acid as control. The experiment ran for 26 days.

Results: A significant increase in the degradation of type I, II, and III collagen (C1M, C2M, and C3M, respectively) was detected on day $22(P=0.0068, P=0.0068, P<0.0001$, respectively), whereas no significant difference in formation (P1NP, P2NP, and P3NP) was detected at any time point ( $P=0.22, P=0.53, P=0.53$, respectively). The CTX-II level increased strongly from disease onset and onwards.

Conclusion: A nearly total separation between diseased and control animals was detected with C3M, making it a good diagnostic marker. The balance of type I, II, and III collagen was significantly altered with CIA in rats, with favour of degradation of the investigated collagens. This indicates unbalanced turnover of the surrounding tissues of the synovial joints, leading to increased pain and degeneration of the synovial joints.
\end{abstract}

Keywords: Collagen balance, Rheumatoid arthritis, Matrix metalloproteinase, Synovial membrane, Cartilage, Connective tissue

\section{Background}

Rheumatoid arthritis (RA) is a chronic inflammatory disease, which primarily affects the extra-cellular matrix (ECM) of the synovial joints [1]. The exact etiology of the disease is still unknown, but several factors such as gender [2], genetics [3,4] and antigens [1] are thought to be involved. The extensive inflammation of the synovial membrane (i.e. synovitis) and other joint tissues induces

\footnotetext{
* Correspondence: aso@nordicbioscience.com

${ }^{1}$ Nordic Bioscience, Herlev, Denmark

Full list of author information is available at the end of the article
}

secretion of proteolytic enzymes leading to degradation of cartilage and elevated bone turnover [5-7].

The synovial membrane is divided into two separate layers, an intimate layer of cells embedded in ECM and a sub-intimae layer of loose connective tissue $[8,9]$. The first layer consists of two types of cells, fibroblast-like synoviocytes and macrophages. These cells form a capsule enclosing the joint, containing type I and III collagen $[9,10]$. In healthy individuals this layers is approximately $1-4$ cells thick, whereas it can be up to 12 cells thick in individuals with RA $[1,8]$. The increased amount of cells results in increased concentrations of degradative enzymes, such as 
matrix metalloproteinases (MMP) and aggrecanases, which are released into the synovial fluid, leading to increased degradation and formation of the surrounding ECM of cartilage, connective tissue, and the synovial membrane itself [11-13]. It is well known that MMP-1, -3, -9 , and -13 is upregulated with RA [13-15]. These MMP's are important proteolytic enzymes in ECM breakdown as MMP-1 degrades type I, II and III collagen, MMP-3 degrades, amongst other ECM proteins, type III collagen and the proteoglycans of cartilage, MMP-9 degrades type IV and V collagens, and MMP-13 degrades type I, II, and III collagen, however type II collagen more efficiently than type I and III collagen.

The protein fragments derived from the proteolytic enzyme cleavage, called neo-epitopes, are potentially released from the tissues and may enter into the circulation and thereby serve as biochemical markers indicating the degree of tissue destruction. There are already several commercial biochemical markers availably utilizing neo-epitopes; CTX-I [16], PIIANP [17], COMP [18], and ICTP [19].

In this study we used newly developed biochemical markers based on neo-epitopes of type I, II, and III collagen degradation (C1M [20], C2M [21], C3M [22]) and formation (P1NP [23], P2NP(unpublished), P3NP(unpublished)) to describe the turnover of cartilage, synovial membrane, and connective tissue. The degradation markers are all derived from cleavage with RA related MMP's and especially C3M have shown to be increased with increasing fibrosis [24-28] and Ankylosing Spondylitis [29]. The formation markers are all measures of the N-terminal pro-collagen, which are also believed to be MMP derived. Moreover, to assess in more detail the degradation of cartilage and to control that the CIA model was working, the well-known CTX-II was used [30].

The aim of this study was to investigate the effect of autoimmunity by collagen induced arthritis (CIA) on type I, II, and III collagen turnover in rats utilizing the newly developed biomarker assays C1M, C2M, C3M, P1NP, P2NP, and P3NP, as well as CTX-II, before arthritis occur and at a late stage of arthritis.

\section{Methods \\ Reagents}

If not stated chemicals and reagents were purchased from Sigma-Aldrich (Copenhagen, Denmark) or VWR (Herlev, Denmark). The ELISA plate, pre-coated with streptavidin (nunc clear 96 well-plates), were purchased from Roche Diagnostics (cat.: 11940279, Mannheum, Germany). All ELISA plates were analyzed with the ELISA reader from Molecular Devices, SpectraMax M, (CA, USA). Rats were purchased from Charles River (Charles River, Germany). Antibodies were produced at
Nordic Bioscience, Beijing. ELISA coating peptides was purchased from American Peptide Company.

\section{Collagen induced arthritis in rats}

CIA was induced in 10, 7 week old, female Lewis rats by immunizing with $450 \mu \mathrm{l} 2 \mathrm{mg} / \mathrm{ml}$ porcine type II collagen dissolved in $0.05 \mathrm{M}$ acetic acid and emulsified 1:1 in Incomplete Freunds Adjuvant on day 0 and 7, as described earlier [30]. 10 Lewis rats only injected with $0.05 \mathrm{M}$ acetic acid was used as control. Every day, starting from day 8 , rats were examined for visual signs of disease, defined as macroscopic evidence of increase in paw size, determined paw score. A score from 0-4 was given depending on the swelling; 0: No evidence of erythema and swelling, 1: Erythema and mild swelling confined to the tarsals or ankle joint, 2: Erythema and mild swelling extending from the ankle to the tarsals, 3: Erythema and moderate swelling extending from the ankle to the metatarsal joints, 4: Erythema and severe swelling encompass the ankle, foot, and digits or ankylosis of the limb. Rats reaching a paw score of 10 were sacrificed, due to too great pain. Moreover, paw volume was measured. Paw volume is a widely used method to detect disease in the CIA model [31,32]. Paw volume increases due to inflammation, which is accompanied by interstitial oedema. We used a simple and accurate method to determine paw volume described before [32], with minor changes. Briefly, a $200 \mathrm{ml}$ beaker with Mill-Q water was placed on a sensitive digital weight (sensitivity: $0.01 \mathrm{~g}$ ). The rat's paws were, one by one, lowered into the water. The sum of difference in weight from baseline for the paws was determined as the arbitrary number of paw size. The animal experiment protocol was approved by the local animal Ethics committee at Nordic Bioscience Beijing. The ethical approval number is NBB-AM-R/ 2009-01.

On day 16 all CIA rats were diseased, determined by paw score. The rats were sacrificed on day 26 , besides if there were extensive paw swelling, determined by paw score, or a too great weight loss $(>10 \%)$, then rats was terminated earlier. Serum samples were collected throughout the experiment from overnight fasted animals.

\section{Quantification of collagens Degradation of type I collagen}

The newly developed C1M ELISA detects degraded type I collagen by MMP-2, -9 , and -13 [20]. The ELISA is based a monoclonal antibody (mAb) recognising the fragment GSPGKDGVRG at position 764 in the mature type I collagen. Briefly, $100 \mu \mathrm{l}$ biotinylated coater peptide was added to a streptavitin coated plate. This was incubated at $20^{\circ} \mathrm{C}$ for $30 \mathrm{~min}$, with agitation. Next, the plate was washed 5 times in washing buffer. Twenty $\mu$ l sample or standard together with $100 \mu \mathrm{l} \mathrm{HRP-labelled} \mathrm{antibody}$ 
was added to the plate. This was incubated at $20^{\circ} \mathrm{C}$ for 1 hour with agitation, followed by washing 5 times in washing buffer. One-hundred $\mu \mathrm{l}$ 3,3,5,5'-tetramethylbenzidine (TMB) was added and incubated for $15 \mathrm{~min}$. in the dark at $20^{\circ} \mathrm{C}$ with agitation. Lastly, $100 \mu \mathrm{l}$ stop buffer $\left(0.1 \% \mathrm{H}_{2} \mathrm{SO}_{4}\right)$ was added, before reading the plate at $540 / 590 \mathrm{~nm}$ on an ELISA reader, SpectraMax M. Technical data on C1M is listed in Table 1.

\section{Formation of type I collagen}

The newly, previously described P1NP ELISA detects formation of type I collagen [23]. The assay is specific of a fragment (PEEYVSPDAEVIG) in the N-terminal propeptide of type I collagen. Briefly, $100 \mu$ l biotinylated coater peptide was added to a streptavidin coated plate and was incubated at $4^{\circ} \mathrm{C}$ for $30 \mathrm{~min}$, with agitation. Next, the plate was washed 5 times in washing buffer. Twenty $\mu \mathrm{l}$ sample or standard together with $100 \mu \mathrm{l}$ HRP-labelled antibody was added to the plate. This was incubated at $4^{\circ} \mathrm{C}$ for 1 hour with agitation, followed by washing 5 times in washing buffer. One-hundred $\mu \mathrm{l}$ TMB was added and incubated for $15 \mathrm{~min}$. in the dark at $20^{\circ} \mathrm{C}$ with agitation. Lastly, $100 \mu \mathrm{l}$ stop buffer $(0.1 \%$ $\mathrm{H}_{2} \mathrm{SO}_{4}$ ) was added, before reading the plate at 540/ $590 \mathrm{~nm}$ on an ELISA reader, SpectraMax M. Technical data on P1NP is listed in Table 1.

\section{Degradation of type II collagen}

The novel, previously described, C2M (aka. CIIM) ELISA [21] detects a MMP-degradation fragment (RDGAAG) at position 1053 in the mature type II collagen. Moreover, the commercial available ELISA, CTX-II, detects degradation of type II collagen. This assay was run accordingly to the manufactures protocol. The protocol for $\mathrm{C} 2 \mathrm{M}$ was briefly; $100 \mu \mathrm{l}$ biotinylated coater peptide was added to a streptavidin coated plate and was incubated at $20^{\circ} \mathrm{C}$ for $30 \mathrm{~min}$, with agitation. Next, the plate was washed 5 times in washing buffer. Twenty $\mu$ l sample or standard together with $100 \mu \mathrm{l}$ HRP-labelled antibody was added to the plate. This was incubated at $4^{\circ} \mathrm{C}$ for overnight with agitation, followed by washing 5 times in washing buffer. One-hundred $\mu \mathrm{l}$ TMB was added and incubated for $15 \mathrm{~min}$. in the dark at $20^{\circ} \mathrm{C}$ with agitation. Lastly, $100 \mu \mathrm{l}$ stop buffer $\left(0.1 \% \mathrm{H}_{2} \mathrm{SO}_{4}\right)$ was added, before reading the plate at $540 / 590 \mathrm{~nm}$ on an ELISA reader, SpectraMax M. Technical data on $\mathrm{C} 2 \mathrm{M}$ is listed in Table 1.

\section{Formation of type II collagen}

The novel P2NP ELISA detects the N-terminal propeptide of type II collagen (unpublished). A mAb was raised against the neo-epitope and an ELISA was made with the neo-epitope (biotinylated) as coater in an ELISA plate coated with streptavidin. The neo-epitope was used as standard [1000 ng/ml-0 ng/ml], to get a quantitative ELISA. Briefly, $100 \mu \mathrm{l}$ biotinylated coater peptide was added to a streptavidin coated plate and was incubated at $20^{\circ} \mathrm{C}$ for $30 \mathrm{~min}$, with agitation. Next, the plate was washed 5 times in washing buffer. Twenty $\mu \mathrm{l}$ sample or standard together with $100 \mu \mathrm{l}$ HRP-labelled antibody was added to the plate. This was incubated at $4^{\circ} \mathrm{C}$ overnight with agitation, followed by washing 5 times in washing buffer. One-hundred $\mu \mathrm{l}$ TMB was added and incubated for $15 \mathrm{~min}$. in the dark at $20^{\circ} \mathrm{C}$ with agitation. Lastly, $100 \mu \mathrm{l}$ stop buffer $\left(0.1 \% \mathrm{H}_{2} \mathrm{SO}_{4}\right)$ was added, before reading the plate at $540 / 590 \mathrm{~nm}$ on an ELISA reader, SpectraMax M. Technical data on P2NP is listed in Table 1.

\section{Degradation of type III collagen}

The C3M ELISA is a newly developed ELISA, which is specific of a MMP-9 degradation fragment (GGPGQPGTEGNK) of mature type III collagen [22]. Shortly, $100 \mu \mathrm{l}$ biotinylated coater peptide was added to a streptavidin coated plate and was incubated at $20^{\circ} \mathrm{C}$ for $30 \mathrm{~min}$, with agitation. Next, the plate was washed 5 times in washing buffer. Twenty $\mu$ l sample or standard together with $100 \mu \mathrm{l}$ HRP-labelled antibody was added to the plate. This was incubated at $20^{\circ} \mathrm{C}$ for 1 hour with agitation, followed by washing 5 times in washing buffer. One-hundred $\mu \mathrm{l}$ TMB was added and incubated for $15 \mathrm{~min}$.

Table 1 Overview of the technical specifications of the ELISA's applied in the experiment with CIA rats

\begin{tabular}{|c|c|c|c|c|c|}
\hline Assay name & Target & Specificity of assay & $\begin{array}{l}\text { Detection range } \\
(\mathrm{ng} / \mathrm{ml})\end{array}$ & $\begin{array}{l}\text { Intra-assay } \\
\text { variation (\%) }\end{array}$ & $\begin{array}{l}\text { Inter-assay } \\
\text { variation (\%) }\end{array}$ \\
\hline $\mathrm{C} 1 \mathrm{M}[20]$ & Degradation of type I collagen & $\begin{array}{l}\text { Neo-epitope generated by } \\
\text { MMP-2, 9, and } 13\end{array}$ & $0.83-500$ & 6.7 & 10.1 \\
\hline $\mathrm{C} 2 \mathrm{M}[21]$ & Degradation of type II collagen & $\begin{array}{l}\text { Neo-epitope generated by } \\
\text { several MMP }\end{array}$ & $0.09-2$ & 3.5 & 7.3 \\
\hline $\mathrm{C} 3 \mathrm{M}[22]$ & Degradation of type III collagen & $\begin{array}{l}\text { Neo-epitope generated by } \\
\text { MMP-9 }\end{array}$ & $0.86-50$ & 2.7 & 3.4 \\
\hline P1NP [23] & Formation of type I collagen & N-terminal pro-peptide & $0-1000$ & n.a. & n.a. \\
\hline P2NP (unpublished) & Formation of type II collagen & N-terminal pro-peptide & $0-1000$ & n.a. & n.a. \\
\hline P3NP (unpublished) & Formation of type III collagen & N-terminal pro-peptide & $0.149-76.3$ & 4.1 & 11.0 \\
\hline
\end{tabular}


in the dark at $20^{\circ} \mathrm{C}$ with agitation. Lastly, $100 \mu \mathrm{l}$ stop buffer $\left(0.1 \% \mathrm{H}_{2} \mathrm{SO}_{4}\right)$ was added, before reading the plate at 540/ $590 \mathrm{~nm}$ on an ELISA reader, SpectraMax M. Technical data on C3M is listed in Table 1.

\section{Formation of type III collagen}

The formation of type III collagen was quantified with the newly developed competitive P3NP assay (unpublished). The assay is specific of the fragment (PTGPQNYSP), which is a fragment in the $\mathrm{N}$-terminal pro-peptide of type III collagen. A mAb was raised against the fragment and an ELISA was developed with the fragment (biotinylated) as coater in an ELISA plate coated with streptavidin. To quantify the formation level, the peptide was used a standard in the range $[1000 \mathrm{ng} / \mathrm{ml}-0 \mathrm{ng} / \mathrm{ml}]$. Briefly, $100 \mu \mathrm{l} \mathrm{bio-}$ tinylated coater peptide was added to a streptavidin coated plate and was incubated at $20^{\circ} \mathrm{C}$ for $30 \mathrm{~min}$, with agitation. Next, the plate was washed 5 times in washing buffer. Twenty $\mu \mathrm{l}$ sample or standard together with $100 \mu \mathrm{l}$ HRP-labelled antibody was added to the plate. This was incubated at $4^{\circ} \mathrm{C}$ overnight with agitation, followed by washing 5 times in washing buffer. One-hundred $\mu \mathrm{lTMB}$ was added and incubated for $15 \mathrm{~min}$. in the dark at $20^{\circ} \mathrm{C}$ with agitation. Lastly, $100 \mu \mathrm{l}$ stop buffer $\left(0.1 \% \mathrm{H}_{2} \mathrm{SO}_{4}\right)$ was added, before reading the plate at $540 / 590 \mathrm{~nm}$ on an ELISA reader, SpectraMax M. Technical data on P3NP is listed in Table 1.

\section{Statistics}

All values are presented as mean \pm standard error of mean (SEM) with 10 replications in each of the groups. Significant difference between mean (day 7 and 22) and the day of first immunization (baseline) was determined using the non-parametric Mann-Whitney $t$-test. Differences were considered statistically significant if $\mathrm{P}<0.05$ and significant level as: ${ }^{*}=\mathrm{P}<0.05,{ }^{* * *}=\mathrm{P}<0.01$, and ${ }^{* * *}$ : $\mathrm{P}<0.001$. The turnover of collagen was calculated by taken the ratio of formation compared to degradation.

\section{Results}

\section{Animal health and disease incidence}

The earliest signs of disease in the CIA group were observed on day 14, measured by increase in paw volume (Figure 1A). By day 16 all immunized rats showed sign of disease (Figure 1C) and this time point was defined as disease onset. Paw volume increased in the diseased rat throughout the experiment, whereas no increase in paw volume was detected in the control group (Figure 1A). In addition, paw score was assessed and a significant difference between the groups was detected from day 15 and until termination of the experiment (Figure 1B). Three animals in the diseased group were sacrificed before the end of the study, due to a paw score higher than 10. The swelling of the paws was detected in the hind paws one to two days before signs of swelling were detected in the front paws. At the late stage, the activity of diseased rats were affected by the swelling, as most of the time, they were not active.

Figure 1D depicts the weight of the animals. The mean weight of the control group followed the normal weight gain for growing rats, with an increase of approximately $12 \%$ during the 3 weeks of the experiment. The mean weight of the CIA rat increased more rapidly until disease onset (day 16), whereafter the weight decreased significantly $(\mathrm{p}<0.001)$ compared to the control group.

\section{Tissue balance of connective tissue, cartilage, and synovial membrane}

The tissue balance of connective tissue was assessed by measurements of degradation and formation of type I collagen. The level of all biochemical markers of collagen is listed in Table 2. A 51.2\% $(\mathrm{P}=0.0068)$ increase in type I collagen degradation $(\mathrm{C} 1 \mathrm{M})$ was detected in serum from diseased animals on a late time point (day 22) compared to baseline level (Figure 2B). No difference in degradation of type I collagen was found between the groups before disease onset (day 7, Figure 2A). A decrease in formation of type I collagen (P1NP) compared to baseline level were detected in both treated and control animals during the experiment, but with no significance between the groups (Figure 2C, D). This imbalanced amount of degradation and formation, led to an overall significant increase in turnover (i.e. ratio between degradation and formation) of type I collagen on day 22 of $169 \%(\mathrm{P}=0.0052)$ in the diseased animals (Figure 2F). No significant difference was detected at the early time point in the control or diseased group (Figure 2E).

Type II collagen was used to determine the tissue balance of cartilage, as type II collagen is the main collagen of cartilage and it is not abundant in any other tissue. Degradation of type II collagen was measured by two different assays, utilizing two different neo-epitopes; the novel C2M ELISA [21] and the well-known CTX-II ELISA [33]. Both C2M and CTX-II showed significantly increased degradation in the diseased animals compared to control at the late time point, $31.9 \%(\mathrm{P}=0.0068)$ and $10963 \%(\mathrm{P}=0.0004)$, respectively (Figure 3B, D). However, CTX-II already showed increased degradation by more than $100 \%$ at disease onset in the treated animals compare to control (data not shown). No change in type II collagen degradation was detected before disease onset (Figure 3A, C). Furthermore, there was no significant difference between the formation of type II collagen (P2NP) in diseased animals compared to baseline or control (Figure 3E, F). Only at the late time point a significant increase $(11765 \%, \mathrm{P}=0.0004)$ of turnover of type II collagen was detected with CTX-II (Figure 3J). 
A

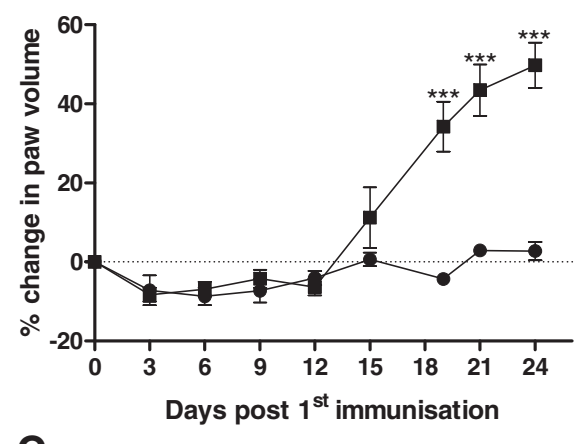

C

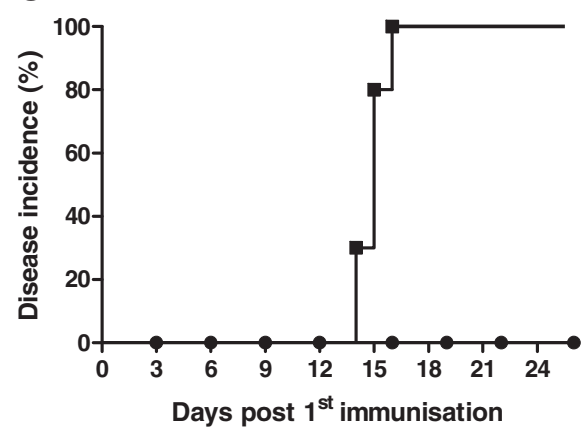

B
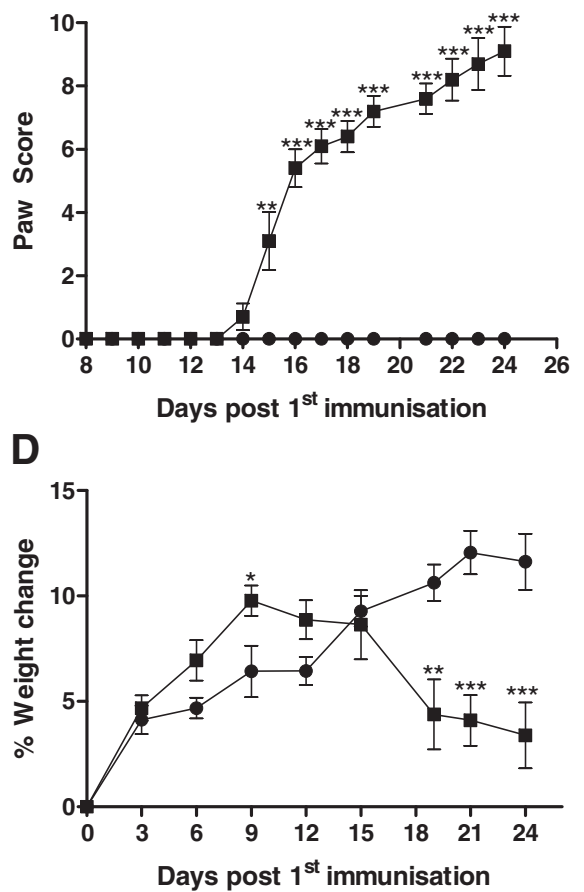

Figure 1 The percentage increase in paw volume (A), paw score (B), percentage disease incidence (C), and percent weight changes (D) observed post $1^{\text {st }}$ immunization. The CIA group (squares, $n=10$ ) and control animals (circles, $n=10$ ) were compared by Mann-Whitney $t$-test. All statistical analyses were calculated based on baseline corrected values. Data are shown as mean \pm SEM and significance levels as *: P<0.05, **: $P<0.01,{ }^{* * *}: P<0.001$.

Table 2 Biochemical markers measured in serum on day 7 and 22 compared to baseline

\begin{tabular}{lllll}
\hline & Time point & Control & CIA & P-value \\
\hline C1M & Day 7 & $9.939 \pm 7.963$ & $15.63 \pm 9.461$ & 0.6038 \\
& Day 22 & $9.283 \pm 7.216$ & $51.2 \pm 8.947$ & $\mathbf{0 . 0 0 6 8} * *$ \\
C2M & Day 7 & $5.376 \pm 10.15$ & $12.39 \pm 11.54$ & 0.5490 \\
& Day 22 & $-7.593 \pm 7.610$ & $31.92 \pm 10.51$ & $\mathbf{0 . 0 0 6 8} * *$ \\
CTX-II & Day 7 & $-38.89 \pm 41.61$ & $-81.54 \pm 6.355$ & 0.7487 \\
& Day 22 & $-87.71 \pm 12.29$ & $10963 \pm 5236$ & $\mathbf{0 . 0 0 0 4} * * *$ \\
C3M & Day 7 & $-1.609 \pm 4.95$ & $4.312 \pm 8.117$ & 0.6608 \\
& Day 22 & $0.4491 \pm 3.92$ & $39.73 \pm 5.447$ & $<\mathbf{0 . 0 0 0 1} * * *$ \\
P1NP & Day 7 & $-14.72 \pm 9.157$ & $-19.59 \pm 13.53$ & 0.4002 \\
& Day 22 & $-20.67 \pm 5.433$ & $35.59 \pm 7.097$ & 0.2176 \\
P2NP & Day 7 & $2.327 \pm 13.29$ & $-4.001 \pm 5.772$ & 0.8633 \\
& Day 22 & $3.346 \pm 11.57$ & $-4.766 \pm 9.643$ & 0.8534 \\
P3NP & Day 7 & $-1.829 \pm 3.588$ & $-8.746 \pm 2.936$ & 0.3562 \\
& Day 22 & $2.334 \pm 2.849$ & $6.045 \pm 2.790$ & 0.5288 \\
\hline
\end{tabular}

The CIA $(n=10)$ and control groups $(n=10)$ were compared by Man-Whitney $\mathrm{t}$-test on both days. All statistical analyses were calculated based on baseline corrected values. Data are shown as mean \pm SEM and significance levels as *: $\mathrm{P}<0.05,{ }^{* *}: \mathrm{P}<0.01,{ }^{* * *}: \mathrm{P}<0.001$.
The tissue balance of connective tissue, including the synovial membrane, was determined by quantification of degradation and formation of type III collagen, the main collagen in the synovial membrane. The degradation of type III collagen (C3M) was increased with 39.7\% $(\mathrm{P}<0.0001)$ at the late time point in the diseased group compared to control (Figure 4B), but no change was detected at the early time point (Figure 4A). There was no significant difference in formation of type III collagen (P3NP) between the groups at the early or late time point (Figure 4C, D). However, a tendency towards elevated formation was detected in the diseased animals compared to baseline on the late time point. Overall, an elevated, but not significant, amount $(17.4 \%, \mathrm{P}=0.24)$ of type III collagen turnover was detected in the diseased rats at the early time point (Figure $4 \mathrm{E}$ ) and a significant increase in turnover was detected at the late time point $(32.4 \%, \mathrm{P}<0.0001$, Figure 4F).

\section{Discussion}

Synovitis is the main characteristic of RA, however, the effect on the tissues involved of the increasing amount of proteolytic enzymes released into the synovial fluid due to the increase in synovial cells have not been studied in detail. Here we have used a CIA rat model to 


\section{A}

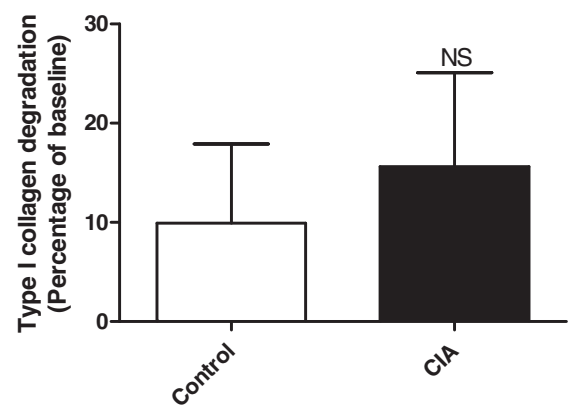

C

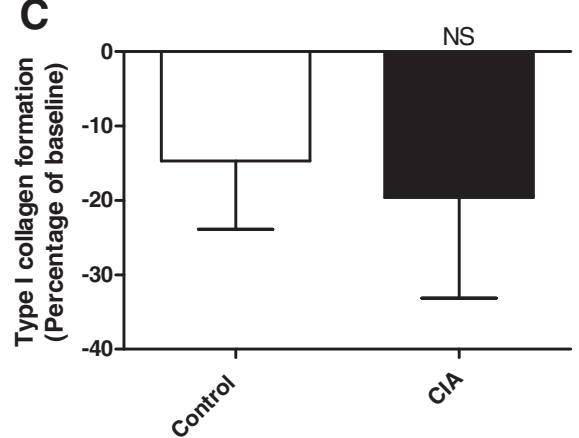

E

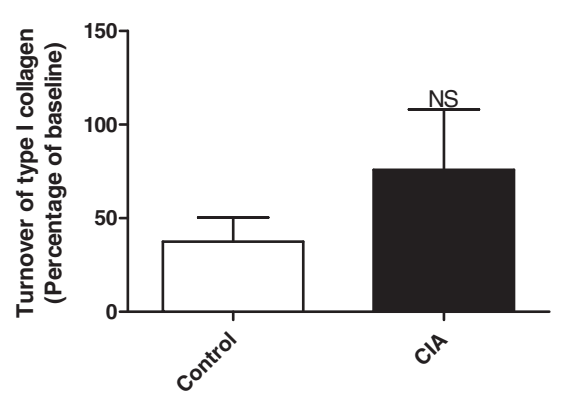

Day 7
B

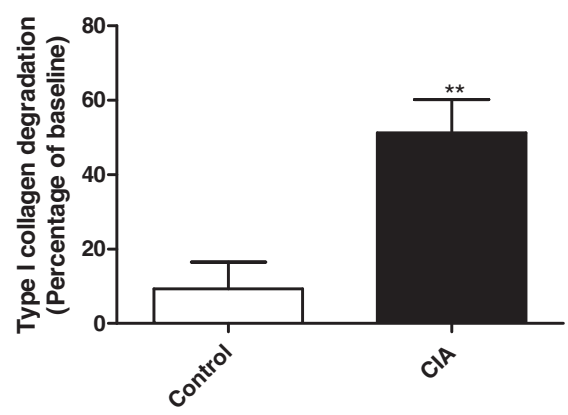

D

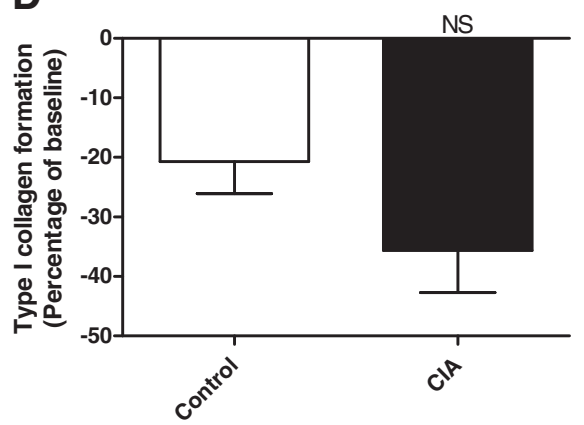

$\mathbf{F}$

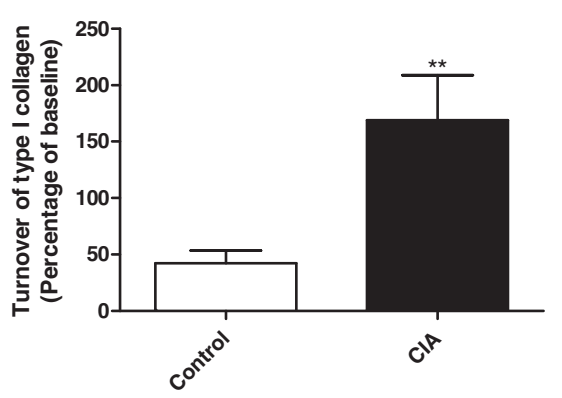

Day 22

Figure 2 Biomarkers of type I collagen turnover on day $\mathbf{7}$ and $\mathbf{2 2}$ corrected to baseline. A, B: Type I collagen degradation detected with C1M. Range of measurement was [55.4-116.7]. C, D: Formation of type I collagen detected with P1NP. Range of measurement was [398.0-8603.1]. E, F: Calculations of type I collagen turnover. Range of measurement was [0.009-0.1]. The CIA group (black, $\mathrm{n}=10$ ) and control animals (white, $\mathrm{n}=10$ ) were compared by Mann-Whitney $t$-test. All statistical analysis was calculated based on baseline corrected values. Data are shown as mean \pm SEM and significance levels as *: $P<0.05,{ }^{* *}: P<0.01,{ }^{* * *}: P<0.001$.

study the release of protein fragments of collagens, derived from cleavage with proteolytic enzymes, specifically MMP's. An increase of degradation of type I, II, and III collagens was found, reflecting an elevated level of turnover of the collagens after disease onset. No significant difference in the formation of the collagens was detected between controls and diseased rats at any time point. In summary, the tissue balance of cartilage, synovial membrane, and connective tissue was disturbed.

The ELISA used to quantify type I collagen MMPdriven turnover (C1M [20], P1NP [23]) is not bone specific as conventional ELISAs of type I collagen, but the neo-epitope of the ELISA derives from connective tissues. C1M have earlier been found to significantly increase in ankylosing spondylitis patients compared to gender and age matched controls [29]. As there was no significant difference in the formation of type I collagen between the groups or compared to baseline in our study, an overall increase in type I collagen turnover was observed. This indicates an altered balance of connective tissue in the CIA rats towards degradation. Type I collagen is the most abundant collagen in vertebrates and is found in scar tissue, skeleton, synovial membrane, skin, tendons, ligaments, artery, etc. Many macromolecules, such as integrins, fibronectin, and decorin bind to type I collagen and certain cells like fibroblast attach to the 


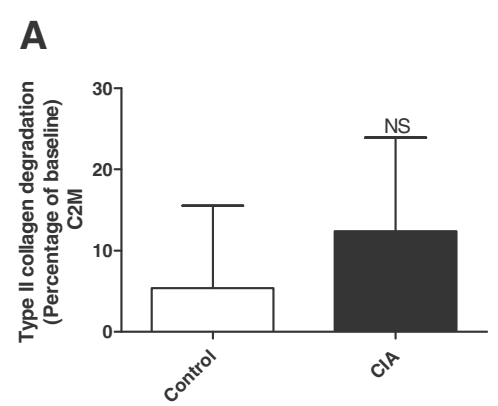

B
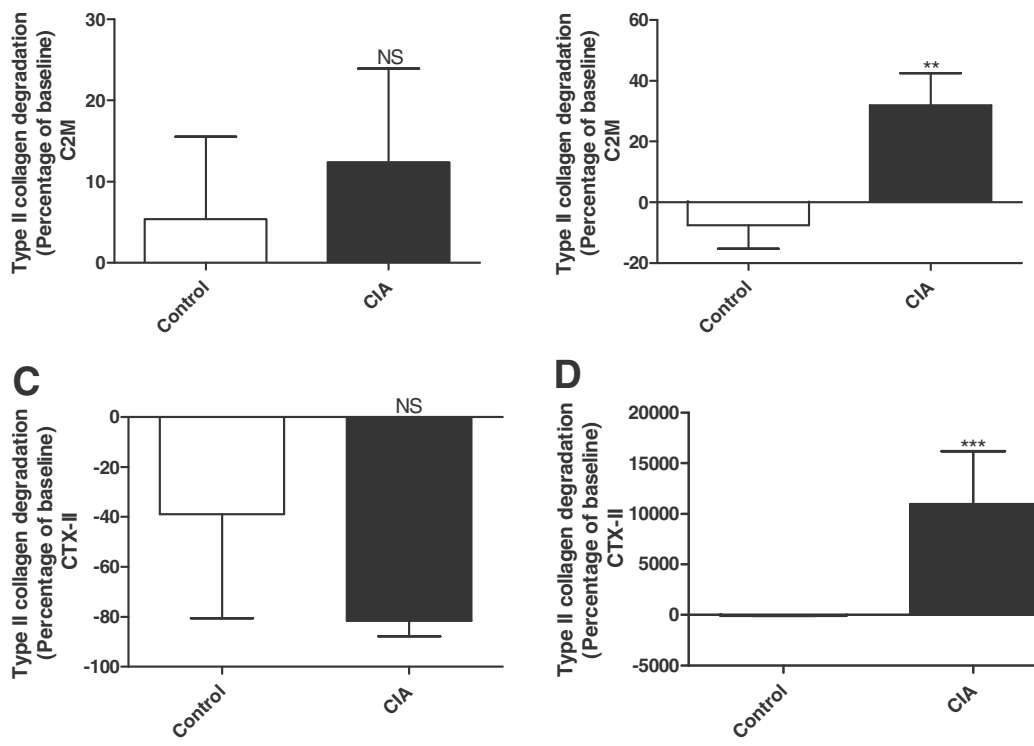

D
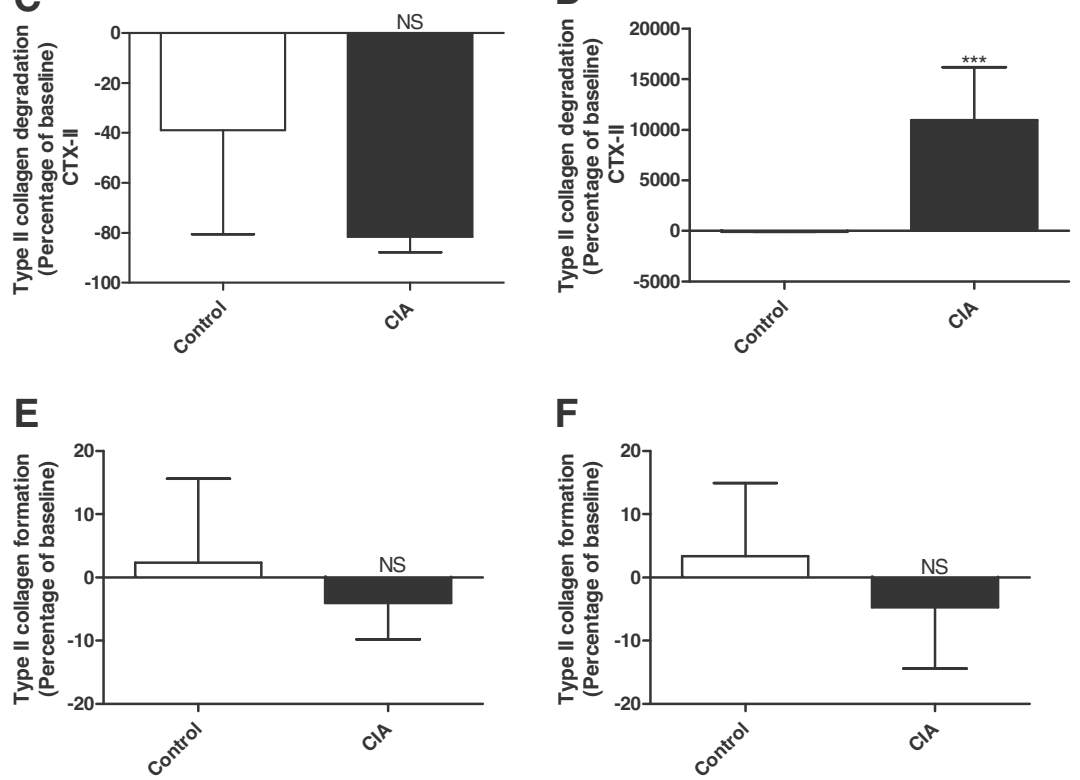

$\mathbf{F}$

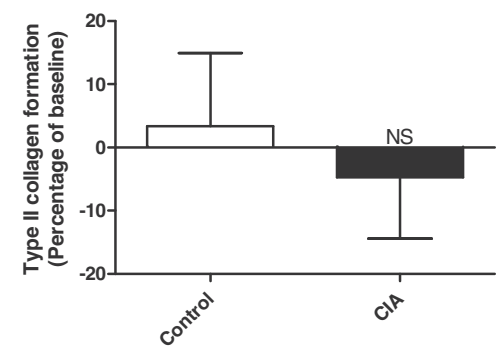

G

H
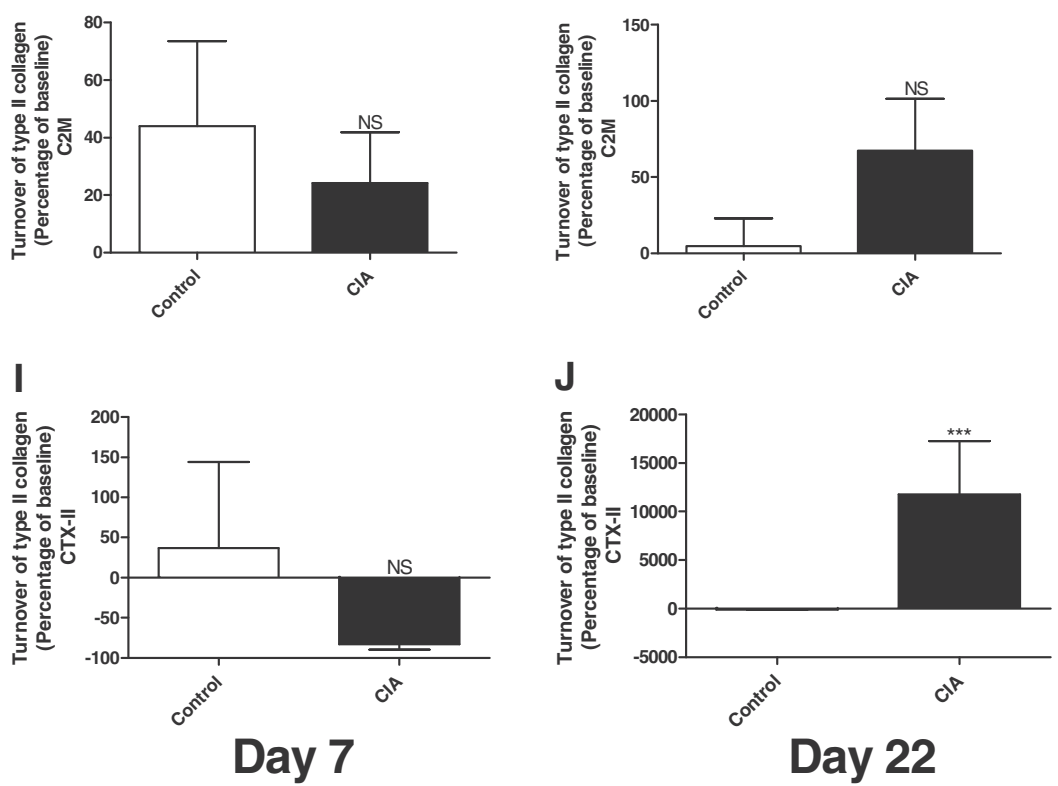

Figure 3 (See legend on next page.) 
(See figure on previous page.)

Figure 3 Biomarkers of type II collagen turnover on day $\mathbf{7}$ and 22 corrected to baseline. A, B: Type II collagen degradation detected with C2M. Range of measurement was [0.3-1.2]. C, D: Type II degradation detected with CTX-II. Range of measurement was [0.2-2132.7]. E + F.

Formation of type II collagen detected with P2NP. Range of measurement was [167.3-877.5]. G, H: Calculations of type II collagen turnover with C2M. Range of measurement was [0.0005-0.006]. I + J: Calculation of type II collagen turnover with CTX-II. Range of measurement was [0.3-72.0]. The CIA group (black, $n=10$ ) and control animals (white, $n=10$ ) were compared by Mann Whitney $t$-test. All statistical analyses were calculated based on baseline corrected values. Data are shown as mean \pm SEM and significance levels as *: $P<0.05,{ }^{* *}: P<0.01, * * * P<0.001$.

ECM protein [34-36]. Therefore, the increased degradation of type I collagen in this experiment could reflect many metabolic processes. It could be that the skeleton and tendons weakens, due to decreased movement because of pain, or the increase in fibroblast in the synovial membrane increase the level of MMP's increasing the degradation of type I collagen in the surrounding bone and synovial membrane. What exactly the degradation of type I collagen reflects in this experiment is still to be determined.

Type II collagen is the most abundant collagen in cartilage and CTX-II have in many studies been used to quantify cartilage degradation [30,37,38]. CTX-II has been shown to increase in RA patients receiving treatment (Cyclosporine, Methotrexate, and/or Steroid) compared to baseline. However, not even after four years of treatment a positive change in cartilage formation was detected, meaning that cartilage balance is compromised even at diagnosis of RA [37]. In the same study Christensen et al. showed that the CTX-II level was correlated to RA activity, demonstrating that the CTX-II level is coupled to synovitis activity [37]. In another study, a reduction in CTX-II level was found after three months in RA patients receiving treatment (prednisolone, Methotrexate, sulfasalazine alone or in combination) [39]. This result conflict with the earlier described study by Christensen et al. [37], where no positive effect of treatment was detected on cartilage. Together, these results could indicate that the right treatment could help the right patient group. However, there is no evidence for this theory yet.

A $250 \%$ increase in cartilage degradation quantified by CTX-II in serum was detected at a late time point in diseased rats in a pre-clinical study of CIA rats. However, an even greater increase (400\%) in degradation was detected between the diseased rats and controls, when quantifying CTX-II in paw extracts [30]. However, alone CTX-II only reflects a part of the mechanisms of cartilage breakdown, suggesting that a panel of biomarkers of type II collagen degradation are to be used to get a broader understanding of mechanisms of cartilage breakdown. In this study we used both C2M and CTXII, which showed relatively different levels of degradation, however the same profile. Both markers of type II collagen was used, not only to get a broader understanding of collagen breakdown, but also because CTX-II was used a control of the CIA model working, as CTX-II have been described in the model previously [30]. C2M is detecting a neo-epitope of type II collagen derived from MMP-cleavage, which is specific of articular cartilage [21], where the neo-epitope in CTX-II is also derived from MMP-cleavage, but it is present during both bone sclerosis and growth plate remodelling [40]. In this experiment the CTX-II fragment was detected to a much higher extend (343 times) than the C2M fragment. This may partly reflect the release of several different MMPs from synovial fibroblasts in the inflamed synovial membrane, which could account for the difference in degradation level of cartilage reflected in this study. A difference in cartilage breakdown, when comparing two biomarkers, has previously been shown with CTX-II and Helix-II [41]. In this study Charni-Ben et al. suggested using various biomarkers reflecting the same metabolic process concurrently to increase the knowledge about the metabolic process. This is true in the case of basal science, but when applied to clinical science, measuring several biomarkers reflecting one metabolic process will be overestimating. In the clinic, a panel of biomarkers reflecting different metabolisms in a given disease gives a broader understanding of the specific case and a better tool to diagnose. Moreover, as both biomarkers of cartilage degradation showed the same profile of cartilage breakdown in our experiment, one biomarker of cartilage degradation is adequate to estimate the state of cartilage balance. As CTX-II is only detectable in human urine and not human serum and $\mathrm{C} 2 \mathrm{M}$ is detectable in both human urine and serum, C2M is a better candidate for use in the clinics, as blood samples are already used to detect C-Reactive Protein and Rheumatoid factor and urine samples will therefore not be needed.

The compromised balance of connective tissue and cartilage turnover is, as stated earlier, a result of the thickened synovial membrane. As RA is an inflammatory disease of the synovial joint, a prognostic RA biomarker panel must include biomarkers to detect the state of the inflamed synovial membrane. This could potentially be used to detect RA in an early stage, i.e. before joint degradation. In this study, we used the turnover of type III collagen, as a marker of the synovial membrane. As the synovial membrane thickness, due to fibrogenesis, an increase in P3NP and no change in C3M were expected. However, a significant $(P>0.0001)$ increase in degradation of type III collagen was 

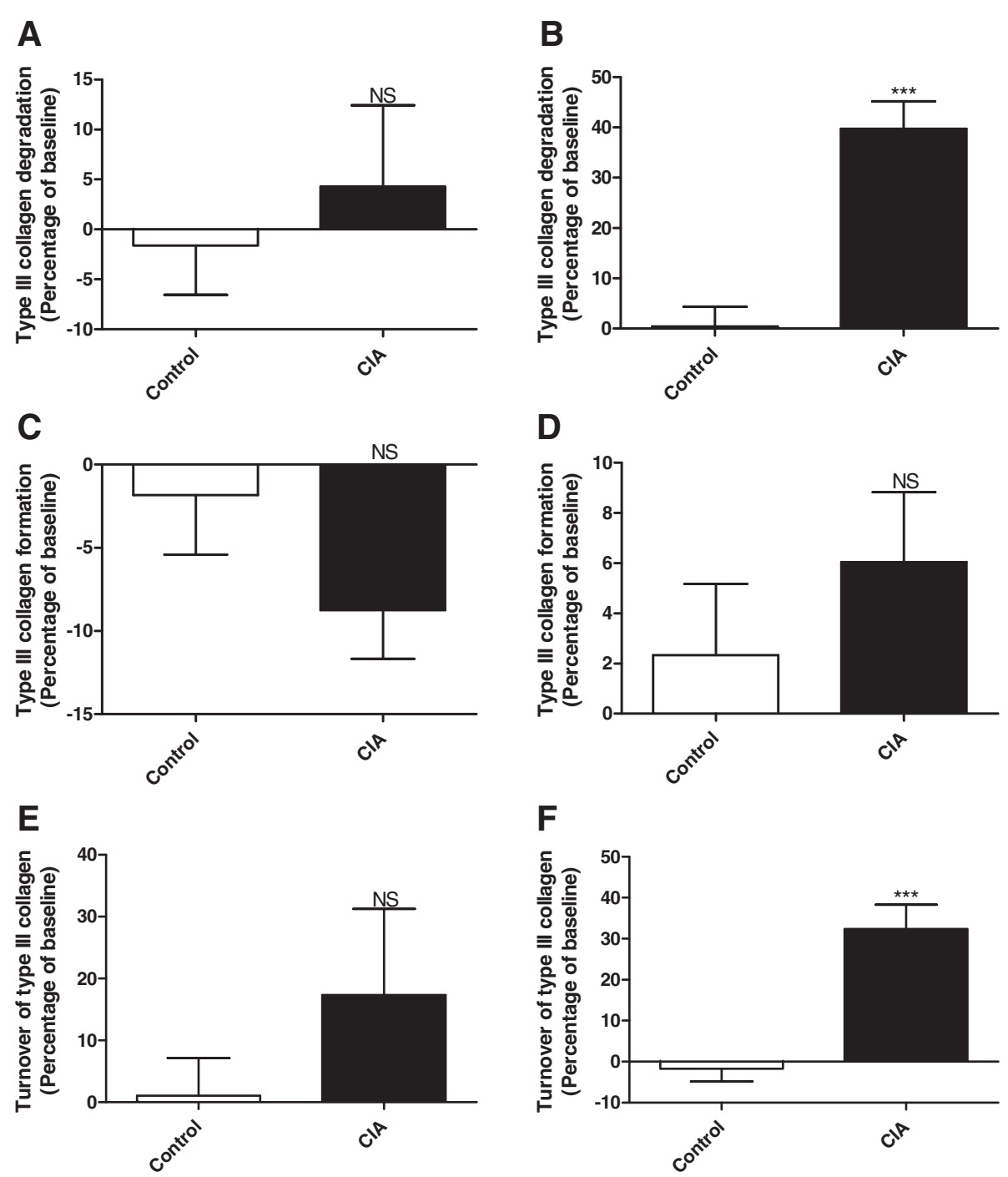

Day 7

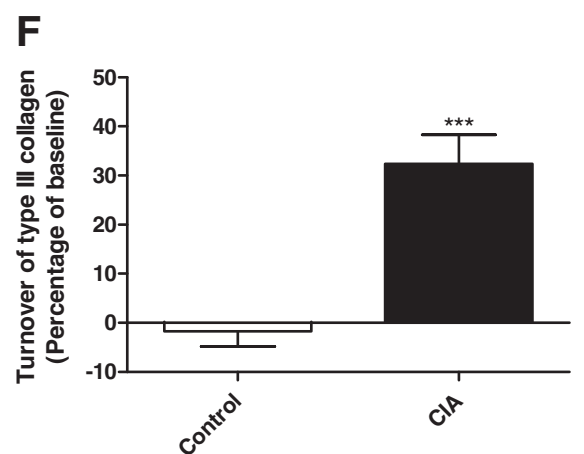

Day 22

Figure 4 Biomarkers of type III collagen turnover on day 7 and 22 corrected to baseline. A, B: Type III collagen degradation detected with C3M. Range of measurement was [2.6-35.5]. C, D: Formation of type III collagen detected with P3NP. Range of measurement was [13.9-71.5]. E, F: Calculations of type III collagen turnover Range of measurement was [0.1-0.6]. The CIA group (black, $n=10$ ) and control animals (white, $n=10$ ) were compared by Mann-Whitneyt-test. All statistical analyses were calculated based on baseline corrected values. Data are shown as mean \pm SEM and significance levels as *: $P<0.05,{ }^{* *}: P<0.01,{ }^{* * *}: P<0.001$.

detected at the late time point, but no significant difference in formation was found between CIA and control rats in this experiment, though a tendency towards increased P3NP in diseased rats was detected at the late time point. This suggests inhibited fibrogenesis in the diseased rats after disease onset and therefore not an increase of synovial membrane thickness. However, the N-terminal propeptide of type III collagen, on which P3NP is based, is not always cleaved of before fibrillation [42]. Therefore, this quantification of formation of type III collagen may be an underestimation and misleading. Moreover, it is very likely that C3M derives from other tissues and not exclusively from the synovial membrane. As the synovial joints swells great quickly in the CIA model, an increase in type III collagen formation (P3NP) was expected, which would correlate to synovial membrane thickness. However, this seems not to be the case.

Type III collagen degradation and formation separately have in several studies been used to detect fibrogenesis [24-28], however this study is the first to combine the biomarkers and investigate tissue balance. In three different fibrosis models (two of liver and one of skin), type III collagen degradation was measured with the C3M ELISA assay. A significantly increase in C3M was detected even after a short disease period in rats compared to control rats [24-26]. However, P3NP is also 
increased in fibrosis, as the amount of tissue is increased. Myllylä et al. [43] found that P3NP was increased in RA patients compared to controls and Hakala et al. [44] even showed that P3NP was increased in RA patients compared to osteoarthritis, psoriasis arthritis, ankylosing spondylitis, and systemic lupus erythematosus patients. Other groups have further enhanced the knowledge that P3NP increases with fibrosis $[27,28]$. To our knowledge P3NP has not been measured along with degradation markers of type III collagen in any murine or human trials, thereby investigating fibrogenesis. We show that fibrogenesis of the synovial membrane is inhibited in CIA rats. As especially C3M has shown to be a possible diagnostic marker of fibrogenesis in rat [24-26] and in Ankylosing Spondylitis patients [29], an investigation if C3M could be an efficacy marker for RA could be interesting. We propose to treat CIA animals with MTX or another RA related drug, to investigate if the level of C3M decreases with treatment. Type III collagen degradation will by itself not be specific of $\mathrm{RA}$, as this has also shown to be elevated in ankylosing spondylitis patients. However, the right combination of collagen biomarkers could be RA specific.

The diagnosis of RA is today based on imaging, selfreported symptoms, and biochemical markers. However, all of these biomarkers and symptoms are only applicable on late stage disease. Therefore, a biomarker panel of early diagnosis of RA is warranted, as this will increase the chances of treatment, maybe even cure. It has been showed that cartilage collagen regeneration is compromised at a very early stage of RA and that this is not primarily inflammation driven [37], increasing the evidence that articular cartilage degradation is a possible early RA biomarker. CTX-II could be one of these early noninvasive biomarkers as CTX-II has been shown to predict joint damage at 4 and 12 weeks in human trials [38]. However, as $\mathrm{C} 2 \mathrm{M}$ is an articular cartilage specific biochemical marker, C2M is a better candidate than CTX-II in an early non-invasive biomarker panel of RA, but more studies confirming it as an early marker is needed. In a measurement of multiple surface proteins (a biomarker panel), Fueldner et al. showed a better discrimination of RA and controls, than when only determining one surface protein [45]. This further increases the evidence of a biomarker panel to be better diagnostic or prognostic than single biomarkers [46]. Moreover, as the pathology of RA involved several cell types, it is reasonable to detect the activation state of more than one cell type.

In this experiment the weight of the rats in the CIA groups was significantly increased before disease onset (day 9). This could be due to water accumulation in the tissues. After disease onset the weight of the diseased rats decrease compared to control rats. This weight loss at the late time point could be because of a decrease in bone and muscle mass, due to reduced activity in the diseased rats. As three rats were sacrificed before experiment termination and the decreased activity of the rats, we propose to use another model of RA, to increase consistency and animal number at experiment termination.

\section{Conclusions}

In summary, we found increased turnover of type I, II, and III collagen, indicating an altered balance of these collagens in RA. The profile of the tissue balances indicates that cartilage, synovial membrane, and connective tissue are important events in RA. The novel C3M biomarker could discriminate between diseased and controls, indicating this as a promising biomarker of synovitis. We speculate that a combination of these biological relevant collagen turnover markers might be utilised in a biomarker panel as diagnostic and prognostic markers of RA.

\section{Abbreviations}

C1M: MMP degraded type I collagen; C2M: MMP degraded type II collagen; C3M: MMP degraded type III collagen; CIA: Collagen induced arthritis; CTXII: C-terminal crosslinked telopeptide type II collagen; ELISA: Enzyme-linked immunosorbent assay; P1NP: N-terminal neo-epitope of type I procollagen; P2NP: N-terminal neo-epitope of type II procollagen; P3NP: N-terminal neoepitope of type III procollagen; RA: Rheumatoid arthritis; TBM: 3,3',5,5tetramethylbenzidine.

\section{Competing interests}

Morten Karsdal is stock holder of Nordic Bioscience.

\section{Authors' contributions}

ASS and AC B-J have designed the experiment. JW, JY and ZQ performed the animal experiment. ASS measured the biochemical markers and did the data analysis. Lastly AC B-J and MK did proofreading. All authors read and approved the final manuscript prior to submission and re-submission.

\section{Author details}

${ }^{1}$ Nordic Bioscience, Herlev, Denmark. ${ }^{2}$ Nordic Bioscience, Beijing, China.

${ }^{3}$ Nordic Bioscience, Beijing, China. ${ }^{4}$ Nordic Bioscience, Beijing, China.

Received: 18 June 2012 Accepted: 17 September 2012

Published: 20 September 2012

\section{References}

1. Buch M, Emery P: The aetiology and pathogenesis of Rheumatoid arthritis. Hospital Parmacist 2002, Ref Type: In Press.

2. Brennan $P$, Silman $A$ : Why the gender difference in susceptibility to rheumatoid arthritis? Ann Rheum Dis 1995, 54:694-695.

3. Macgregor AJ, et al: Characterizing the quantitative genetic contribution to rheumatoid arthritis using data from twins. Arthritis Rheum 2000, 43:30-37.

4. Deighton CM, Wentzel J, Cavanagh G, Roberts DF, Walker DJ: Contribution of inherited factors to rheumatoid arthritis. Ann Rheum Dis 1992, 51:182-185.

5. Rousseau JC, et al: Patients with rheumatoid arthritis have an altered circulatory aggrecan profile. BMC Musculoskelet Disord 2008, 9:74

6. Keyszer G, et al: Circulating levels of matrix metalloproteinases MMP-3 and MMP-1, tissue inhibitor of metalloproteinases 1 (TIMP-1), and MMP1/TIMP-1 complex in rheumatic disease. Correlation with clinical activity of rheumatoid arthritis versus other surrogate markers. J Rheumatol 1999, 26(2):251-258

7. Mamehara A, et al: Serum matrix metalloproteinase-3 as predictor of joint destruction in rheumatoid arthritis, treated with non-biological disease modifying anti-rheumatic drugs. Kobe J Med Sci 2010, 56:E98-E107.

8. Smith MD: The normal synovium. Open Rheumatol J 2011, 5:100-106. 
9. Eyre DR, Muir H: Type III collagen: A major constituent of rheumatoid and normal human synovial membrane. Connect Tissue Res 1975, 4:11-16.

10. Lovell CR, Nicholls AC, Jayson MI, Bailey AJ: Changes in the collagen of synovial membrane in rheumatoid arthritis and effect of D-penicillamine. Clin Sci Mol Med 1978, 55:31-40.

11. Weiss JB, et al: Occurrence of type III collagen in inflamed synovial membranes: a comparison between non rheumatoid, rheumatoid, and normal synovial collagens. Biochem Biophys Res Commun 1975, 65:907-912.

12. Konttinen $Y T$, et al: Analysis of 16 different matrix metalloproteinases (MMP-1 to MMP-20) in the synovial membrane: different profiles in trauma and rheumatoid arthritis. Ann Rheum Dis 1999, 58:691-697.

13. Kim KS, et al: Expression levels and association of gelatinases MMP-2 and MMP-9 and collagenases MMP-1 and MMP-13 with VEGF in synovial fluid of patients with arthritis. Rheumatol Int 2011, 31:543-547.

14. Pap T, et al: Differential expression pattern of membrane-type matrix metalloproteinases in rheumatoid arthritis. Arthritis Rheum 2000, 43:1226-1232

15. Lee EG, et al: Ethyl acetate fraction from Cudrania tricuspidata inhibits IL1beta-induced rheumatoid synovial fibroblast proliferation and MMPs, COX-2 and PGE2 production. Biol Res 2010, 43:225-231.

16. Bonde $M$, et al: Measurement of bone degradation products in serum using antibodies reactive with an isomerized form of an 8 amino acid sequence of the C-telopeptide of type I collagen. J Bone Miner Res 1997, 12:1028-1034

17. Rousseau JC, et al: Serum levels of type IIA procollagen amino terminal propeptide (PIIANP) are decreased in patients with knee osteoarthritis and rheumatoid arthritis. Osteoarthritis Cartilage 2004, 12(6):440-7.

18. Smith RK, Heinegard D: Cartilage oligomeric matrix protein (COMP) levels in digital sheath synovial fluid and serum with tendon injury. Equine Vet $J$ 2000, 32:52-58.

19. Melkko J, Niemi S, Risteli L, Risteli J: Radioimmunoassay of the carboxyterminal propeptide of human type I procollagen. Clin Chem 1990, 36:1328-1332.

20. Leeming $D$, et al: A novel marker for assessment of liver matrix remodeling: an enzyme-linked immunosorbent assay (ELISA) detecting a MMP generated type I collagen neo-epitope (C1M). Biomarkers 2011, 16:616-628.

21. Bay-Jensen AC, et al: Enzyme-linked immunosorbent assay (ELISAs) for metalloproteinase derived type II collagen neoepitope, CIIM-increased serum CIIM in subjects with severe radiographic osteoarthritis. Clin Biochem 2011, 44:423-429.

22. Barascuk N, et al: A novel assay for extracellular matrix remodeling associated with liver fibrosis: An enzyme-linked immunosorbent assay (ELISA) for a MMP-9 proteolytically revealed neo-epitope of type III collagen. Clin Biochem 2010, 43:899-904.

23. Leeming DJ, et al: Enzyme-linked immunosorbent serum assays (ELISAs) for rat and human $\mathrm{N}$-terminal pro-peptide of collagen type I (PINP)-assessment of corresponding epitopes. Clin Biochem 2010, 43:1249-1256

24. Vassiliadis E, et al: Measurement of CO3-610, a Potential Liver Biomarker Derived from Matrix Metalloproteinase-9 Degradation of Collagen Type III, in a Rat Model of Reversible Carbon-Tetrachloride-Induced Fibrosis. Biomark Insights 2011, 6:49-58.

25. Veidal SS, et al: Matrix metalloproteinase-9-mediated type III collagen degradation as a novel serological biochemical marker for liver fibrogenesis. Liver Int 2010, 30:1293-1304.

26. Vassiliadis $\mathrm{E}$, et al: Measurement of matrix metalloproteinase 9-mediated collagen type III degradation fragment as a marker of skin fibrosis. BMC Dermatol 2011, 11:6.

27. Lin DY, Chu CM, Sheen IS, Liaw YF: Serum carboxy terminal propeptide of type I procollagen to amino terminal propeptide of type III procollagen ratio is a better indicator than each single propeptide and $7 \mathrm{~S}$ domain type IV collagen for progressive fibrogenesis in chronic viral liver diseases. Dig Dis Sci 1995, 40:21-27

28. Ulrich D, Noah EM, Burchardt ER, Atkins D, Pallua N: Serum concentration of amino-terminal propeptide of type III procollagen (PIIINP) as a prognostic marker for skin fibrosis after scar correction in burned patients. Burns 2002, 28:766-771.

29. Bay-Jensen AC, et al: Ankylosing spondylitis is characterized by an increased turnover of several different metalloproteinase-derived collagen species: a cross-sectional study. Rheumatol Int 2011.
30. Nielsen $\mathrm{RH}$, Christiansen C, Stolina M, Karsdal MA: Oestrogen exhibits type II collagen protective effects and attenuates collagen-induced arthritis in rats. Clin Exp Immunol 2008, 152:21-27.

31. Bolon B, et al: Rodent preclinical models for developing nove antiarthritic molecules: comparative biology and preferred methods for evaluating efficacy. J Biomed Biotechnol 2011, 2011:569068.

32. Fereidoni M, Ahmadiani A, Semnanian S, Javan M: An accurate and simple method for measurement of paw edema. J Pharmacol Toxicol Methods 2000, 43:11-14.

33. Immunodiagnostic Systems Inc. Method; 2012. Ref Type: Electronic Citation.

34. Trojanowska M, LeRoy EC, Eckes B, Krieg T: Pathogenesis of fibrosis: type 1 collagen and the skin. J Mol Med (Berl) 1998, 76:266-274.

35. Jokinen J, et al: Integrin-mediated cell adhesion to type I collagen fibrils. J Biol Chem 2004, 279:31956-31963.

36. Dzamba BJ, Wu H, Jaenisch R, Peters DM: Fibronectin binding site in type I collagen regulates fibronectin fibril formation. J Cell Biol 1993, 121:1165-1172.

37. Christensen AF, et al: Uncoupling of collagen II metabolism in newly diagnosed, untreated rheumatoid arthritis is linked to inflammation and antibodies against cyclic citrullinated peptides. J Rheumatol 2010, 37:1113-1120

38. Karsdal MA, et al: Biochemical markers of ongoing joint damage in rheumatoid arthritis-current and future applications, limitations and opportunities. Arthritis Res Ther 2011, 13:215

39. Landewe $\mathrm{R}$, et al: Markers for type II collagen breakdown predict the effect of disease-modifying treatment on long-term radiographic progression in patients with rheumatoid arthritis. Arthritis Rheum 2004 50:1390-1399

40. Bay-Jensen AC, et al: Biochemical markers of type II collagen breakdown and synthesis are positioned at specific sites in human osteoarthritic knee cartilage. Osteoarthritis Cartilage 2008, 16:615-623.

41. Charni-Ben TN, et al: The type II collagen fragments Helix-II and CTX-II reveal different enzymatic pathways of human cartilage collagen degradation. Osteoarthritis Cartilage 2008, 16:1183-1191.

42. Jensen LT: The aminoterminal propeptide of type III procollagen. Studies on physiology and pathophysiology. Dan Med Bull 1997, 44:70-78.

43. Myllyla R, Becvar R, Adam M, Kivirikko Kl: Markers of collagen metabolism in sera of patients with various rheumatic diseases. Clin Chim Acta 1989, 183:243-252.

44. Hakala M, et al: Application of markers of collagen metabolism in serum and synovial fluid for assessment of disease process in patients with rheumatoid arthritis. Ann Rheum Dis 1995, 54:886-890.

45. Fueldner $C$, et al: Identification and evaluation of novel synovial tissue biomarkers in rheumatoid arthritis by laser scanning cytometry. Arthritis Res Ther 2012, 14:R8.

46. Syversen SW, et al: Biomarkers in early rheumatoid arthritis: longitudinal associations with inflammation and joint destruction measured by magnetic resonance imaging and conventional radiographs. Ann Rheum Dis 2010, 69:845-850.

doi:10.1186/1479-5876-10-195

Cite this article as: Siebuhr et al: Matrix Metalloproteinase-dependent turnover of cartilage, synovial membrane, and connective tissue is elevated in rats with collagen induced arthritis. Journal of Translational Medicine 2012 10:195

\section{Submit your next manuscript to BioMed Central and take full advantage of:}

- Convenient online submission

- Thorough peer review

- No space constraints or color figure charges

- Immediate publication on acceptance

- Inclusion in PubMed, CAS, Scopus and Google Scholar

- Research which is freely available for redistribution 